\title{
Endoscopic resection of posterior nasal nerves for management of severe allergic rhinitis with intractable nasal hyper-reactivity
}

\author{
Sachio Takeno, MD, PhD 1), Daisuke Takahara, MD 1), Takashi Ishino, MD, PhD 1), \\ Mark Wake, FRCS, MD 2) \\ 1) Department of Otolaryngology, Head and Neck Surgery, Hiroshima University, Hiroshima, Japan, \\ 2) ENT Department, Leicester Royal Infirmary
}

\section{Background and Objectives}

Unregulated nasal hypersensitivity due to pathological neural networks surrounding the inferior turbinate may be responsible for refractory symptoms in severe allergic rhinitis (AR). Posterior nasal neurectomy (PNN) is a novel endoscopic treatment which may be appropriate when standard medical treatments prove ineffective (Fig.1). This prospective study assessed the results of this procedure (1) and used nasal nitric oxide (NO) as an objective measurement of allergic inflammation.

\section{Patients and Methods}

We analyzed 30 patients who underwent PNN surgery. The procedure was performed by selective resection of the posterior nasal nerves with preservation of the sphenopalatine artery (Fig. 2). Symptom scores were evaluated according to the Japanese guidelines for allergic rhinitis. Fractional exhaled NO (FeNO) levels were recorded orally and nasally; pre-treatment, 6 and 12 months following surgery as described elsewhere (2).

\section{Results}

Our endoscopic technique used purpose-built micro instruments which allowed access and selective resection of the nerve branches with preservation of the sphenopalatine artery (SPA). Mean nasal symptom scores were significantly decreased after 12 months $\quad(p<0.0001)$. In addition, $27 \%(8 / 30)$ of patients remained almost symptom free without medication. Concurrently, nasal FeNO levels were significantly reduced at 6-month and 12-month post-treatment (Fig. 3).

\section{Discussion}

The posterior nerve bundles consist of parasympathetic and sympathetic components of the vidian nerve and somatosensory fibers from the maxillary branch of the trigeminal nerve. Herein, we introduce a technique to dissect the nerve bundle and to selectively resect isolated nerve branches, keeping the adjacent SPA intact. Regarding clinical effectiveness, most of the patients subjectively reported excellent or good results. Nasal NO measurements are considered to be an objective marker for AR due to augmented activity of NO metabolism in the inferior turbinate. This study also supports the applicability of nasal NO as an objective outcome parameter for clinical intervention trials in AR.

\section{Conclusions}

This minimally invasive technique appears to be clinically effective and we found that it influences nasal NO metabolism (which is responsible for sustaining allergic inflammation).

\section{Refferences}

1) Ogawa T, Takeno S, et al. Auris Nasus Larynx 2007; 34: 319-326.

2) Takeno S, et al. Auris Nasus Larynx 2017;44: 435-441.

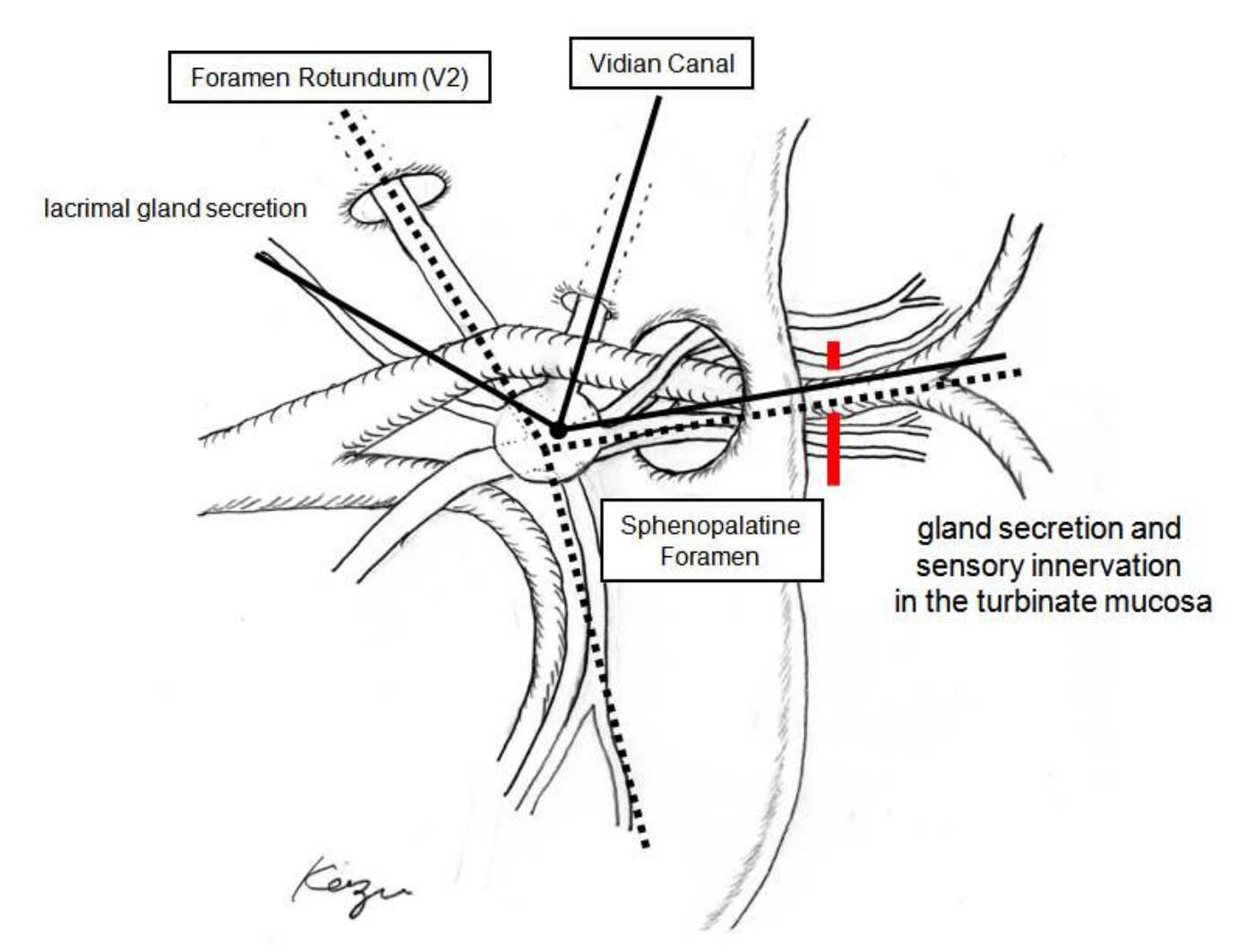

Fig. 1 Schematic illustration of the pterygopalatine fossa with its neural and vascular components through the sphenopalatine foramen. Solid lines: autonomic innervation. Dashed lines: sensory innervation.

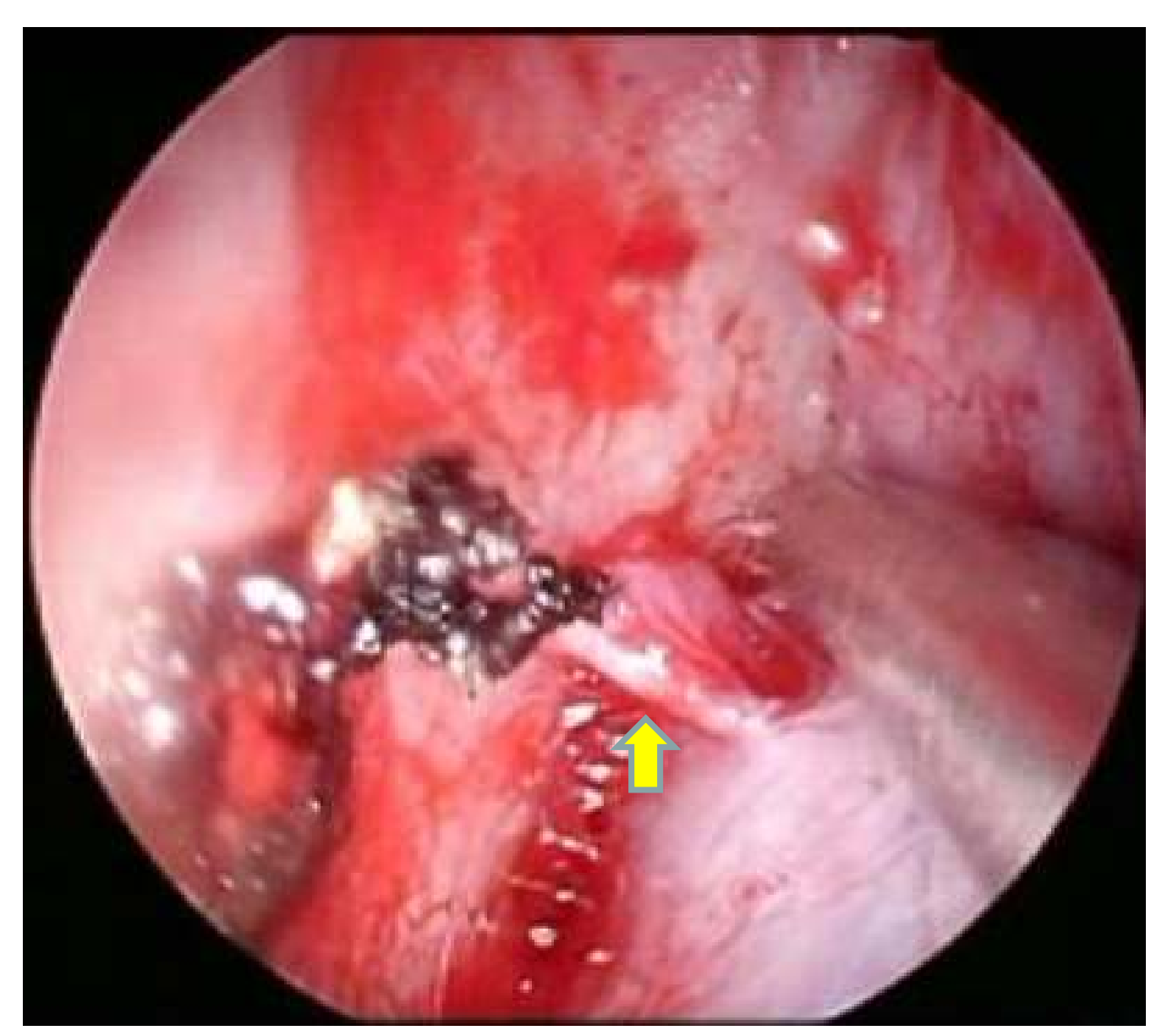

Fig. 2 Intraoperative endoscopic view showing the branch of the posterior nasal nerve (arrow) emerging from the SPF. (a) total nasal symptom scores

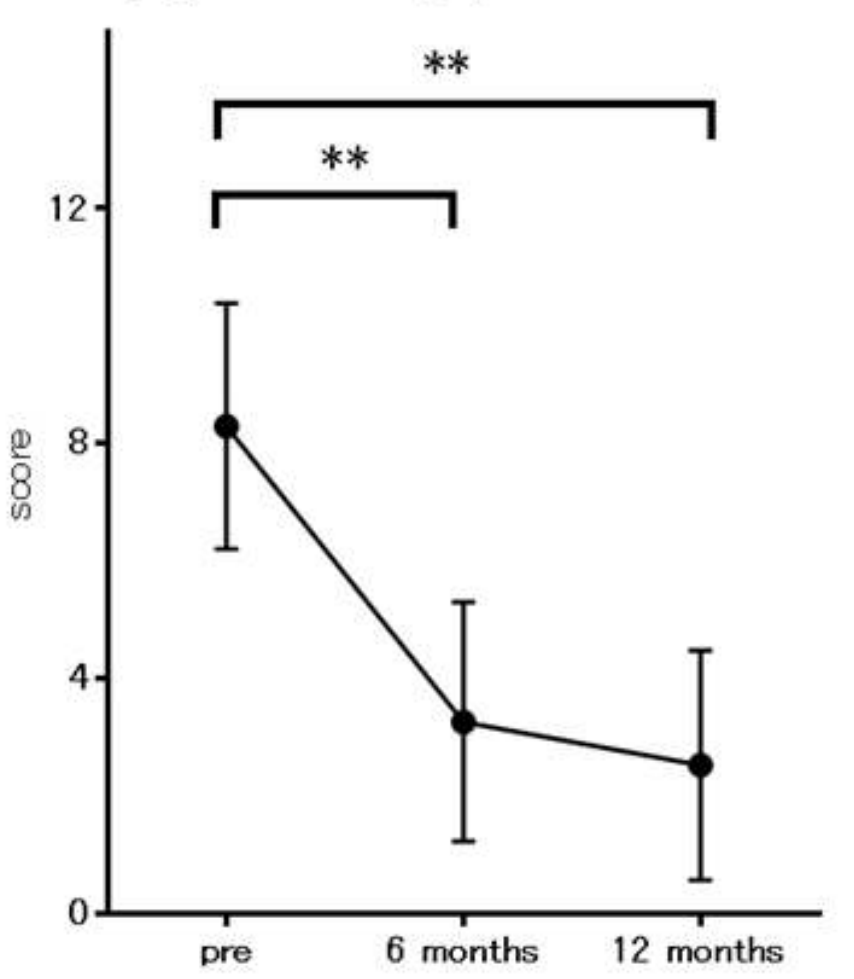

(b)

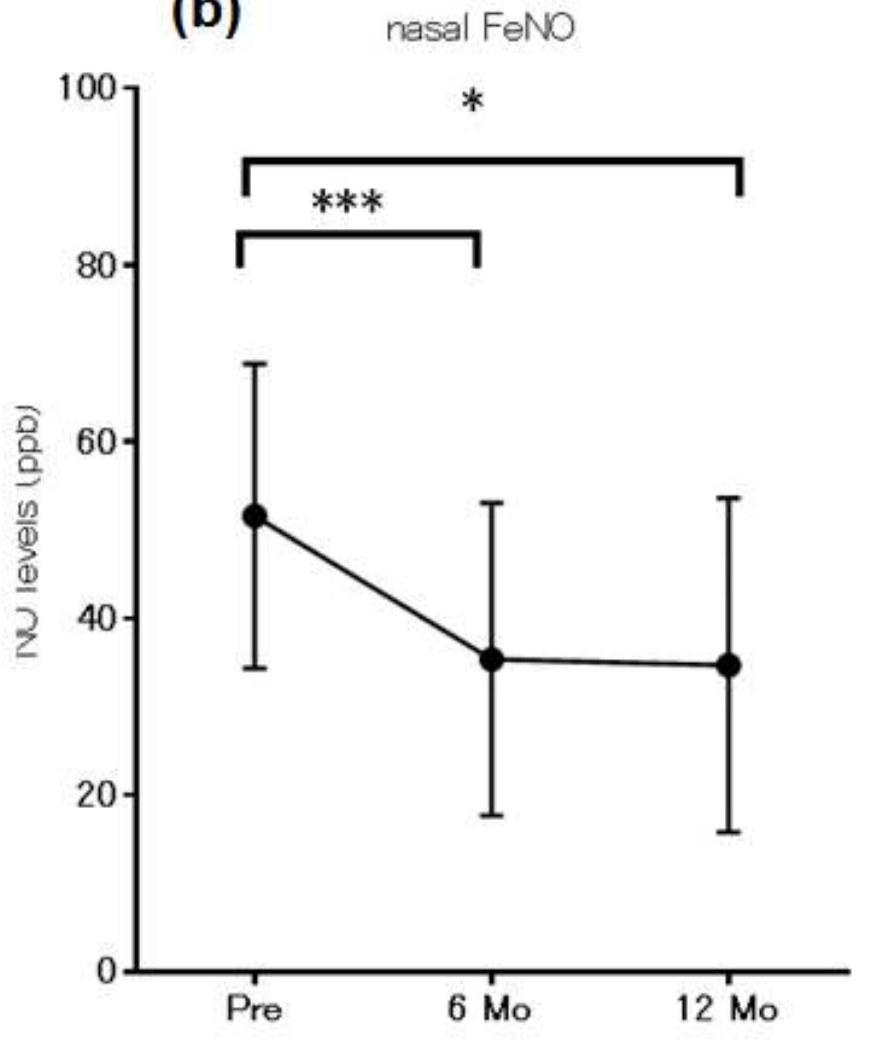

Fig. 3 Time course changes in (a) the total nasal symptom score (TNSS) and (b) nasal FeNO levels. Symbols and error bars represent mean values with standard errors. Pre and post-treatment results are shown; ${ }^{*} p<0.05 ;{ }^{* *} p<0.01 ;{ }^{* *} p<0.001$. 